\title{
ОБ ОДНОЙ СПЕКТРАЛЬНОЙ ЗАДАЧЕ В ПЛОСКОМ УГЛЕ ДЛЯ СИНГУЛЯРНОГО ЭЛЛИПТИЧЕСКОГО ДИФФЕРЕНЦИАЛЬНОГО ОПЕРАТОРА ВТОРОГО ПОРЯДКА
}

\author{
Н. В. Королев, А. А. Ларин \\ (Статьл представлена членом редакиионной коллегии С. М. Ситником) \\ ВУНЦ ВВС «ВВА им. проф. Н. Е. Жуковского и Ю. А. Гагарина», \\ г. Воронеж, 394064, Россия \\ E-mail: korolevn33@yandex.ru, dohior@yandex.ru
}

\begin{abstract}
Аннотация. В работе решена спектральная задача специального вида для сингулярного эллиптического оператора второго порядка в неограниченном угловом секторе на плоскости. Показана перестройка собственных значений и изменение формы собственных функций при вариации угла раствора сектора. Установлена необходимость изменения граничных условий при трансформации области поиска решения от углового сектора к полуплоскости.
\end{abstract}

Ключевые слова: $B$-эллиптическое уравнение, собственные значения, собственные функции, задача Штурма-Лиувилля.

Для цитирования: Королев Н. В., Ларин А. А. 2020. Об одной спектральной задаче в плоском угле для сингулярного эллиптического дифференциального оператора второго порядка. Прикладная математика \& Физика, 52(2): 86-92. DOI 10.18413/2687-0959-2020-52-2-86-92.

\section{ON A SPECTRAL PROBLEM IN THE PLANE ANGLE FOR SECOND ORDER SINGULAR DIFFERENTIAL OPERATOR}

\author{
N. V. Korolev, A. A. Larin \\ (Article submitted by a member of the editorial board C. M. Sitnik) \\ MESC AF «N. E. Zhukovsky and Y. A. Gagarin Air Force Academy», \\ Voronezh, 394064, Russia \\ E-mail: korolevn33@yandex.ru, dohior@yandex.ru \\ Received April 21, 2020
}

\begin{abstract}
In this paper the spectral problem of a special form for the singular elliptic operator of the second order in an unbounded angular sector on a plane is solved. The rearrangement of eigenvalues and changing the shape of eigenfunctions with variations in the sector angle is shown. The necessity of changing the boundary conditions during the transformation of the solution search area from an angular sector to a half-plane is established.

Key words: $B$-elliptic equation, eigenvalues, eigenfunctions, Sturm-Liouville problem.

For citation: Korolev N. V., Larin A. A. 2020. On a spectral problem in the plane angle for second order singular differential. Applied Mathematics \& Physics, 52(2): 86-92 (in Russian).

DOI 10.18413/2687-0959-2020-52-2-86-92.
\end{abstract}

1. Введение. Начиная с работы [Келдыш, 1951], в теории дифференциальных уравнений с частными производными сформировалось направление, в работах которого изучаются краевые задачи для сингулярных и вырождающихся эллиптических уравнений. Специфика постановок таких задач определяется как наличием особенностей в коэффициентах уравнений [Киприянов, 1997], так и допустимым поведением искомого решения в рассматриваемой области [Ландис, 1971; Катрахов, Ситник, 2018]. Последнее замечание относится даже к уравнениям с гладкими коэффициентами [Катрахов, Ситник, 2018]. Требования, предъявляемые к решению, такие как, например, его ограниченность, определяют особенность постановки соответствующих краевых условий. Примером такой задачи, в которой ищутся непрерывные, а потому локально ограниченные решения сингулярного эллиптического уравнения, подчиненные некоторому нелокальному условию, служит задача для стационарного уравнения Шредингера с кулоновским центрально-симметричным потенциалом. Актуальность подобной задачи в последнее время обусловлена исследованиями в области оптических свойств низкоразмерных систем, в которых достигается квантование энергетических 
уровней носителей заряда хотя бы в одном направлении [Klingshirn, 2005]. В частности, особый интерес связан с процессами оптического поглощения и люминесценции в квантовых точках [Klimov, 2010; Gavrilenko, 2011], в том числе с участием донорно-акцепторных пар [Ovchinnikov et al., 2016; Mora-Ramos et al., 2020]. Известно, что форма таких объектов существенно меняет спектральные характеристики носителей заряда. Однако подобная вариация формы может приводить к необходимости формулировки новых граничных условий с целью сохранения корректности постановки математической задачи или возникновению особых точек в решении, для которых требуется его регуляризация. Ввиду актуальности математических исследований, проводимых при решении упомянутых задач, в данной работе изучается спектральная задача в плоском угле для сингулярного эллиптического оператора второго порядка, содержащего по переменной $y$ дифференциальный оператор Бесселя $B_{y}=\partial^{2} / \partial y^{2}+k \partial /(y \partial y)$ [Киприянов, 1997].

2. Постановка задачи. Пусть $K$ - угол в полуплоскости $E_{2}^{+}=\left\{(x, y) \in E_{2}: y>0\right\}$ с вершиной в точке $O=O(0,0)$, границу которого образует замкнутый луч $Г$ и положительная часть оси $O x$. Обозначим эту часть оси через $\Gamma_{0}$ и пусть $\omega \in(0, \pi)$ - раствор угла $K$. Предполагаем, что $K-$ открытое множество, так что $\bar{K}=K \cup \Gamma \cup \Gamma_{0}$ (черта сверху обозначает замыкание множества в $E_{2}$ ).

В классе функций $C^{2}(K) \cap C^{1}\left(K \cup \Gamma_{0}\right) \cap C(\bar{K})$ будем рассматривать спектральную задачу вида

$$
\begin{gathered}
-\frac{\partial^{2} u}{\partial x^{2}}-\frac{\partial^{2} u}{\partial y^{2}}-\frac{k}{y} \frac{\partial u}{\partial y}-\frac{E}{r} u=\lambda u, \quad(x, y) \in K, \\
\left.u\right|_{\Gamma}=0, \\
\left.\frac{\partial u}{\partial y}\right|_{\Gamma_{0}}=0 .
\end{gathered}
$$

В уравнении (1) $E$ и $k$ - положительные постоянные, $k>1 ; r=\sqrt{x^{2}+y^{2}}$. Помимо граничных условий $(2)$ и (3) подчиним функцию $u(x, y)$ нелокальному условию

$$
\int_{K} u^{2}(x, y) y^{k} d y d x<\infty .
$$

Рассматриваемая спектральная задача имеет изолированные отрицательные собственные значения. Требуется найти эти собственные значения и отвечающие им собственные функции.

3. Решение краевой задачи. Следуя [Никифоров, Уваров, 1984; Титчмарш, 1961], воспользуемся методом разделения переменных. Для этого перейдем в задаче (1)-(3) к полярной системе координат по формулам $x=r \cos \theta, y=r \sin \theta, r \geq 0,0 \leq \theta \leq \omega$ и, полагая $v(r, \theta)=u(r \cos \theta, r \sin \theta)$, получим следующую краевую задачу для непрерывной функции $v(r, \theta)$ в полуполосе $\Pi=\{(r, \theta)$ : $0 \leq r<\infty, 0 \leq \theta \leq \omega\}$ :

$$
\begin{gathered}
\frac{\partial^{2} v}{\partial r^{2}}+\frac{k+1}{r} \frac{\partial v}{\partial r}+\frac{1}{r^{2}}\left(\frac{\partial^{2} v}{\partial \theta^{2}}+k \cot \theta \frac{\partial v}{\partial \theta}\right)+\left(\lambda+\frac{E}{r}\right) v=0, \quad r>0, \quad 0<\theta<\omega, \\
\left.v(r, \theta)\right|_{\theta=\omega}=0, \quad r \geq 0, \\
\left.\frac{\partial v}{\partial \theta}\right|_{\theta=0}=0, \quad r>0, \\
\int_{\Pi} v^{2}(r, \theta) r^{k+1} \sin ^{k} \theta d r d \theta<\infty .
\end{gathered}
$$

Разделение переменных в задаче (4)-(7) по формуле $v(r, \theta)=R(r) \Phi(\theta)$ приводит к спектральным задачам для функций $R(r)$ и $\Phi(\theta)$. Для угловой компоненты $\Phi(\theta)$ получаем задачу ШтурмаЛиувилля вида

$$
\begin{gathered}
-\frac{d^{2} \Phi}{d \theta^{2}}-k \cot \theta \frac{d \Phi}{d \theta}=\mu \Phi, \quad 0<\theta<\omega, \\
\left.\frac{d \Phi}{d \theta}\right|_{\theta=0}=\Phi(\omega)=0 .
\end{gathered}
$$

Каждому фиксированному $\mu$ будут соответствовать радиальные компоненты, которые являются решениями задачи

$$
\frac{d^{2} R}{d r^{2}}+\frac{k+1}{r} \frac{d R}{d r}+\left(\lambda+\frac{E}{r}-\frac{\mu}{r^{2}}\right) R=0, \quad 0<r<\infty
$$




$$
\lim _{r \rightarrow 0} R(r) \text { существует и конечен, } \int_{\Pi} R^{2}(r) r^{k+1} d r<\infty .
$$

Решение задачи (8)-(10) получено в [Ларин, Кириллов, 2017]. Пусть $P_{s}^{q}(t)$ - присоединенная функция Лежандра первого рода аргумента $t$, определенная на разрезе [Бейтмен, Эрдейи, 1973], и пусть $\alpha_{l}$ - положительные решения уравнения

$$
f(\alpha)=P_{\alpha+(k-1) / 2}^{(1-k) / 2}(\cos \omega)=0,
$$

которые будем считать занумерованными в порядке возрастания $l=1,2, \ldots$ [Гобсон, 1931]. Тогда собственные значения (C3) задачи имеют вид $\mu_{l}=\alpha_{l}\left(\alpha_{l}+k\right)$, а отвечающие им собственные функции $(\mathrm{C} \Phi)$, с точностью до постоянного множителя, записываются в виде

$$
\Phi_{l}(\theta)=(\sin \theta)^{(1-k) / 2} P_{\alpha_{l}+(k-1) / 2}^{(1-k) / 2}(\cos \theta) .
$$

Заметим, что формулой (17) функции $\Phi_{l}(\theta)$ определяются только для $\theta \in(0, \omega]$. Доопределяя их в точке $\theta=0$ по непрерывности, т. е. полагая $\Phi_{l}(0)=\lim _{\theta \rightarrow 0} \Phi_{l}(\theta)$, получим систему функций, принадлежащих классу $C^{2}[0, \omega]$ и удовлетворяющих граничным условиям (10).

Для решения спектральной задачи (11)-(12) с указанными С3 $\mu=\mu_{l}$ фиксируем произвольный индекс $l$ и перейдем к новой функции $S(r)=r^{(k+1) / 2} R(r)$. Краевая задача для $S(r)$ имеет вид

$$
\begin{gathered}
\frac{d^{2} S}{d r^{2}}+\frac{4 \lambda r^{2}+4 E r+1-\left(2 \alpha_{l}+k\right)^{2}}{4 r^{2}} S=0, \quad r>0, \\
\lim _{r \rightarrow 0} S(r) r^{-(k+1) / 2} \text { существует и конечен, } \int_{\Pi} S^{2}(r) d r<\infty .
\end{gathered}
$$

Решение (18) ищем в виде [Никифоров, Уваров, 1984]

$$
S(r)=r^{\alpha_{l}+(k+1) / 2} \exp (-\sqrt{-\lambda} r) y(r),
$$

где $y(r)$ - новая неизвестная функция, для которой получаем уравнение гипергеометрического типа

$$
r \frac{d^{2} y}{d r^{2}}+\left(1+2 \alpha_{l}+k-2 \sqrt{-\lambda} r\right) \frac{d y}{d r}+\left(E-\sqrt{-\lambda}\left(2 \alpha_{l}+k+1\right)\right) y=0 .
$$

Условия (19) обеспечивают применимость теоремы о разрешимости гипергеометрического уравнения [Никифоров, Уваров, 1984], из которой следует, что СЗ $\lambda$ исходной задачи, являющиеся параметром в уравнении (21), находятся из соотношения

$$
E-\sqrt{-\lambda}\left(2 \alpha_{l}+k+1\right)=2 n \sqrt{-\lambda}
$$

так что

$$
\lambda=\lambda_{n, l}=-\frac{E^{2}}{\left(2 n+2 \alpha_{l}+k+1\right)^{2}}, \quad n=0,1,2, \ldots
$$

Нетривиальные решения $y_{n, l}$ уравнения (21) определяются формулой Родрига [Никифоров, Уваров, 1984]

$$
y_{n, l}=\frac{C_{n, l}}{r^{2 \alpha_{l}+k}} \exp \left(\frac{2 E}{2 n+2 \alpha_{l}+k+1} r\right) \frac{d^{n}}{d r^{n}}\left(r^{n+2 \alpha_{l}+k} \exp \left(-\frac{2 E}{2 n+2 \alpha_{l}+k+1} r\right)\right),
$$

где $C_{n, l}$ - произвольные ненулевые постоянные. Из соотношений (20) и (23), а также тождества $R(r)=r^{(k+1) / 2} S(r)$, получаем, что радиальные составляющие $R(r)$ решения $v(r, \theta)$, отвечающие фиксированному $l$, имеют вид

$$
R_{n, l}=\frac{C_{n, l}}{r^{\alpha_{l}+k}} \exp \left(\frac{2 E}{2 n+2 \alpha_{l}+k+1} r\right) \frac{d^{n}}{d r^{n}}\left(r^{n+2 \alpha_{l}+k} \exp \left(-\frac{2 E}{2 n+2 \alpha_{l}+k+1} r\right)\right),
$$

где $n=0,1,2, \ldots$ Таким образом, доказано следующее утверждение.

Теорема 1 Отрицательные собственные значения задачи (1)-(3) определяются равенством (22), а отвечающие им собственные функции в полярной системе координат записываются в виде

$$
u_{n, l}(r \cos \theta, r \sin \theta)=R_{n, l}(r) \Phi_{l}(\theta), \quad l=1,2, \ldots, \quad n=0,1, \ldots
$$

где функиии $R_{n, l}(r)$ и $\Phi_{l}(\theta)$ определяются равенствами (24) и (17), соответственно. 
Отметим, что можно рассматривать подобную задачу и в предельном случае, когда $\omega=\pi$, т. е. в полуплоскости. В этом случае участок границы Г переходит в замкнутый луч $\{(x, 0): x \leq 0\}$, краевое условие $(2)$ отсутствует, а граничное условие $(3)$ ставится на всей прямой $y=0$ с удаленной из нее точкой $O(0,0)$.

При переходе к полярной системе координат условие (5) отсутствует, а условие (6) принимает вид

$$
\left.\frac{\partial v}{\partial \theta}\right|_{\theta=0}=\left.\frac{\partial v}{\partial \theta}\right|_{\theta=\pi}=0, \quad r>0
$$

Новое условие (22) для функции $v(r, \theta)$ приводит к новому граничному условию в задаче $(8)-(10)$ для функции $\Phi(\theta)$. Оно принимает вид $\Phi^{\prime}(0)=\Phi^{\prime}(\pi)=0$. Собственные значения $\mu$ определяются последовательностью $\mu=\mu_{l}=l(l+k)$, где $l=0,1, \ldots$, а соответствующие этим значениям собственные функции с точностью до постоянного множителя имеют вид

$$
\Phi_{l}(\theta)=(\sin \theta)^{(1-k) / 2} P_{l+(k-1) / 2}^{(1-k) / 2}(\cos \theta)
$$

Отметим, что собственную функцию (23) представляют в этом случае алгебраические многочлены от аргумента $\cos \theta$ [Ларин, Кириллов, 2017].

C3 задачи на полуплоскости определяются последовательностью

$$
\lambda=\lambda_{n, l}=-\frac{E^{2}}{(2 n+2 l+k+1)^{2}}, \quad n=0,1,2, \ldots
$$

а радиальные компоненты, отвечающие функции $\Phi_{l}(\theta)$ в представлении для функции $v(r, \theta)$, имеют вид

$$
R_{n, l}=\frac{C_{n, l}}{r^{l+k}} \exp \left(\frac{E}{2 n+2 l+k+1} r\right) \frac{d^{n}}{d r^{n}}\left(r^{n+2 l+k} \exp \left(-\frac{2 E}{2 n+2 l+k+1} r\right)\right)
$$

4. Обсуждение результатов. Рассмотрим спектр краевой задачи на полуплоскости $\theta \in[0, \pi]$ с граничными условиями (22). С3, определяемые формулой (24), представлены на рис. 1. Здесь и далее считаем, что $E=1$ и $k=2$, а также $C_{n, l}=1$. Как видно, все С3, кроме первого, являются кратными со степенью кратности $\gamma=n+1$. С ростом $l$ спектр задачи сгущается, а сами значения стремятся к нулю.

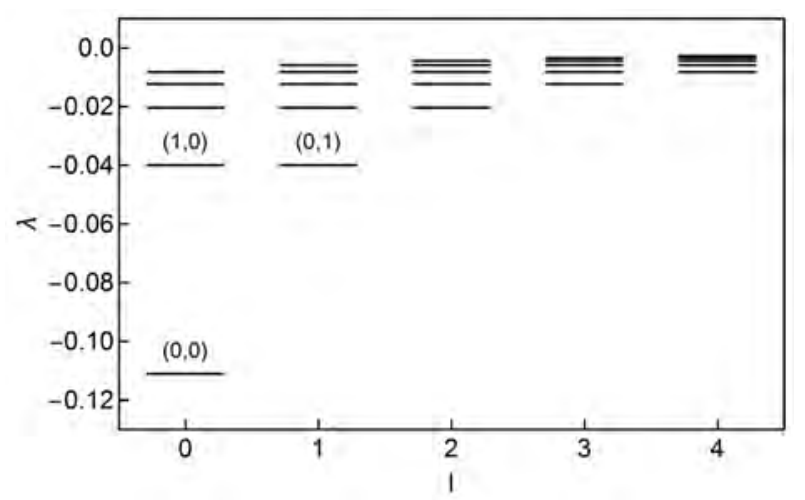

Рис. 1. Собственные значения (24) для различных пар чисел $(n, l)$

Fig. 1. Eigenvalues (24) for various pairs numbers $(n, l)$

Для задачи в неограниченном угле на плоскости $\theta \in[0, \omega]$ картина несколько меняется. Характерное сгущение С3 и их стремление к нулю с ростом $l$ остается аналогичным задаче на полуплоскости, однако для произвольного угла $\omega$ вырождение С3 снимается. Только в частном случае $\omega=\pi / 2 \mathrm{C} 3$ снова становятся вырожденными со степенью $\gamma=n+1$, но в отличие от полуплоскости меняется сочетание индексов $(n, l)$ кратных корней. Например, если $n=2$, то кратными будут С3 с индексами $(1,1)$ и $(0,3)$. При $n=3$ кратными СЗ будут $(2,1),(1,3),(0,5)$. На рис. 2 представлены серии С3 при $l=1, \ldots, 5$ в случае $\omega=11 \pi / 12$. Так, к примеру, для соседних серий СЗ выполняется неравенство $\lambda_{1, l}>\lambda_{0, l+1}$. 


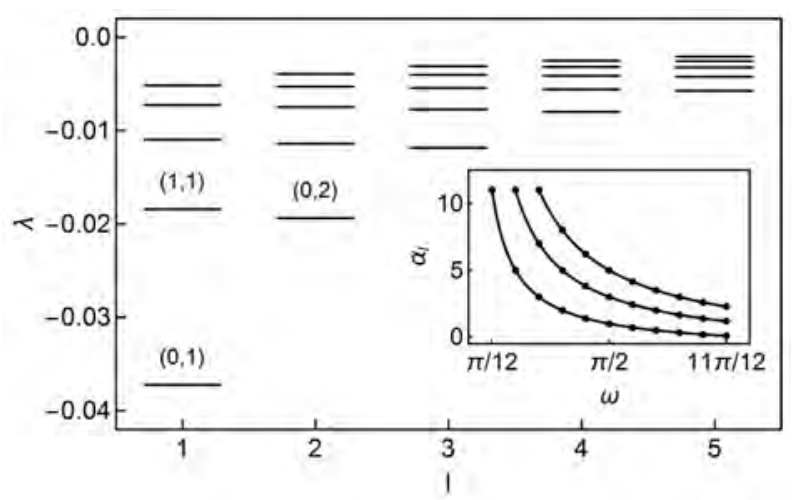

Рис. 2. Собственные значения (22) при различных парах чисел $(n, l)$ для $\omega=11 \pi / 12$. Вставка: зависимость корней $\alpha_{l}$ уравнения (13) от угла $\omega$

Fig. 2. Eigenvalues (22) for various pairs numbers $(n, l)$ at $\omega=11 \pi / 12$. Insert: roots $\alpha_{l}$ of the equation (13) as function of angle $\omega$
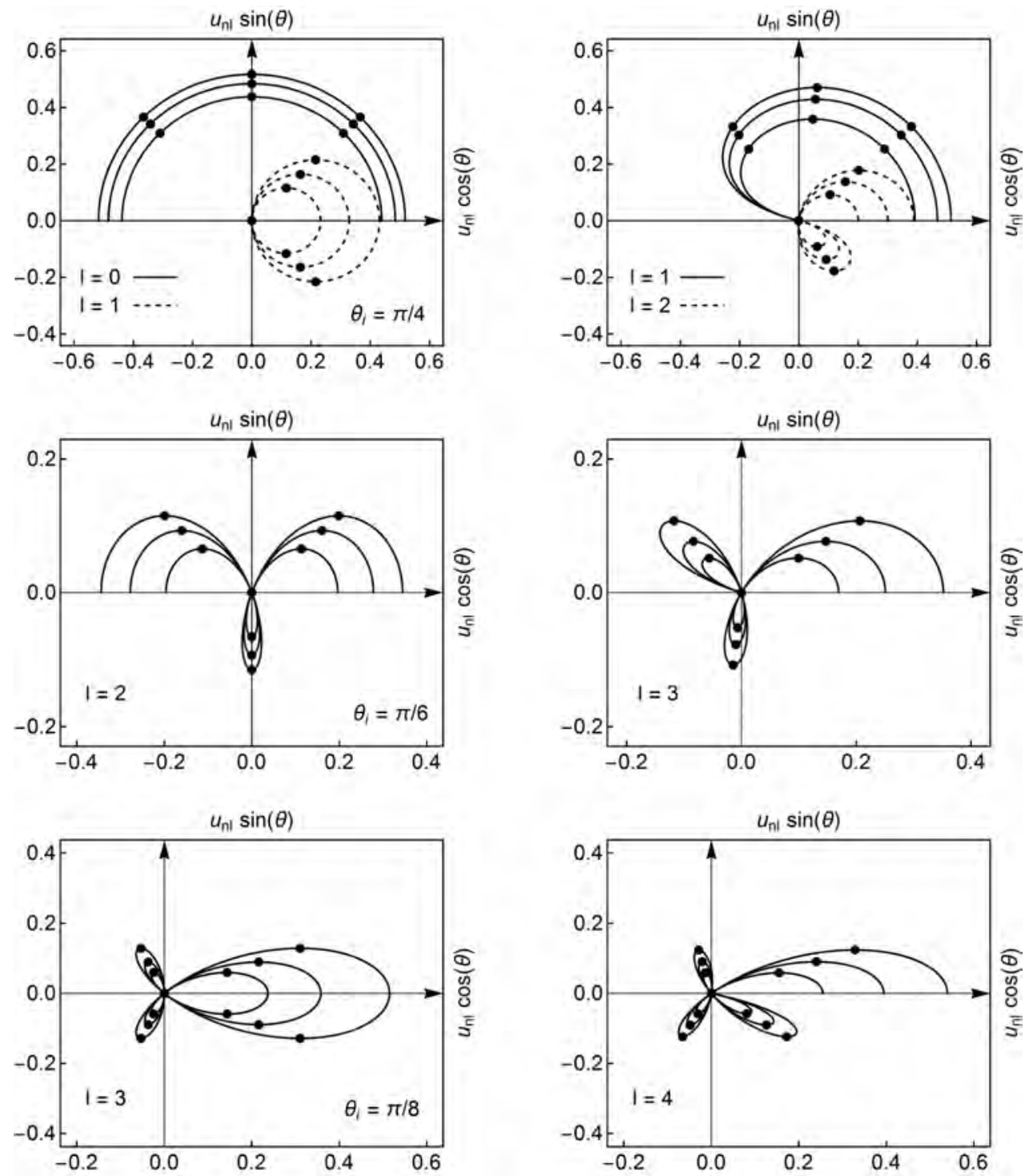

Рис. 3. График функции $u(r \cos \theta, r \sin \theta)$ при различных значениях $l$ на полуплоскости $\omega=\pi($ слева) и при угле $\omega=11 \pi / 12$ (справа)

Fig. 3. Function $u(r \cos \theta, r \sin \theta)$ for various $l$ on the half-plane $\omega=\pi$ (left) and $\omega=11 \pi / 12$ (right)

Поскольку С3 зависят от корней уравнения (13), т. е. зависят от величины угла $\omega$, было выполнено численное решение уравнения (13) при различных $\omega$. Указанные зависимости представлены на вставке к рис. 2 в случаях $l=1,2,3$. Оказывается, что с ростом $\omega$ значения $\alpha_{l}$ убывают как функция $l \pi / \omega-1$, а значит $\alpha_{1} \rightarrow 0$ при $\omega \rightarrow \pi$. В результате приходим к спектру (24). Данная зависимость 
указывает на естественность перехода от граничного условия на луче Г (5) к условию (22). Вид СФ $u(r \cos \theta, r \sin \theta)$ определяется угловой компонентой $\Phi_{l}(\theta)$. Графики СФ в полярной системе координат при $n=0$ представлены на рис. 3 в диапазоне изменения $r=0.20, \ldots, 0.90$. Слева представлены СФ для задачи в полуплоскости $\omega=\pi$, справа для задачи с углом $\omega<\pi$. Точками обозначены узлы сетки при фиксированном угле $\theta_{i}$. Форма СФ зависит от четности $l$. Начиная со значений $l \geq 2$, рост $l$ приводит к увеличению числа петель на графике в области начала координат, в то время как изменение $n$ влияет только на абсолютное значение функции. Для задачи в неограниченном угле симметрия СФ нарушается (рис. 3, справа). Графики представлены при изменении $\theta \in[0,11 \pi / 12]$. Как и в случае с собственными значениями, СФ в пределе при $\omega \rightarrow \pi$ по форме переходят к виду, полученному для задачи на полуплоскости при $\omega=\pi$.

4. Заключение. В работе выполнено исследование спектральной задачи для сингулярного эллиптического дифференциального оператора второго порядка, содержащего дифференциальное выражение Бесселя $B_{y}$. Для двумерного случая рассмотрены угловые секторы при угле раствора $\omega=\pi$ и $0<\omega<\pi$. Показано, что спектр задачи является вырожденным только в случаях $\omega=\pi / 2$ и $\omega=\pi$, однако очередность кратных корней может различаться. Численное решение уравнения с присоединенными функциями Лежандра позволило установить асимптотику СЗ при $0<\omega<\pi$, которая указывает на предельный переход спектра рассматриваемого уравнения при $\omega \rightarrow \pi$. Получены собственные функции и продемонстрировано их изменение при вариации числа $l$.

Таким образом, в рамках поставленной задачи установлено, что для сохранения корректности постановки краевой задачи для сингулярного уравнения при переходе от задачи в угле раствором $\omega<\pi$ к задаче в полуплоскости необходимо изменение граничного условия Дирихле для луча $Г$ на условие Неймана.

\section{Список литературы}

1. Бейтмен Г., Эрдейи А. 1973. Высшие трансцендентные функции Т. 1. М., Наука, 296.

2. Гобсон Е. В. 1952. Теория сферических и эллипсоидальных функций. М., ИЛ, 476.

3. Катрахов В. В., Ситник С. М. 2018. Метод операторов преобразования и краевые задачи для сингулярных эллиптических уравнений. Современная математика. Фундаментальные направления, 64(2): 211-426.

4. Келдыш М. В. 1951. О некоторых структурах вырожденных уравнений эллиптического типа на границе области. Доклады Академии Наук СССР, 77(2): 181-183.

5. Киприянов И. А. 1997. Сингулярные эллиптические краевые задачи. М., Наука. Физматлит, 208.

6. Ландис Е. М. 1971. Уравнения второго порядка эллиптического и параболического типов. М., Наука, 288.

7. Ларин А. А., Кириллов В. П. 2017. Задача на собственные значения для одного обыкновенного дифференциального оператора с сингулярным коэффициентом. Сборник трудов Х международной конференции «ПМТУКТ-2017» (Воронеж, 18-24 сентября 2017 г.). Воронеж, Изд-во Научная книга, 221-225.

8. Никифоров А. Ф., Уваров В. Б. 1984. Специальные функции математической физики. М., Наука, 344.

9. Титчмарш Э. Ч. 1961. Разложения по собственным функциям, связанные с дифференциальными уравнениями второго порядка Ч. 2. М., ИЛ, 554.

10. Gavrilenko V. I. 2011. Optics of Nanomaterials. Singapore, Pan Stanford Publishing, 326.

11. Klingshirn C. 2005. Semiconductors Optics. Berlin, Springer-Verlag Heidelberg, 797.

12. Klimov V. I. 2010. Nanocrystal quantum dots. New York, CRC Press, 453.

13. Mora-Ramos M. E., Aouami A. El., Feddi E., Radu A., Restrepo R. L., Vinasco J. A., Morales A. L., Duque C. A. 2020. Donor impurity energy and optical absorption in spherical sector quantum dots. Heliyon, 6: e03194(11 pp).

14. Ovchinnikov O. V., Smirnov M. S., Korolev N. V., Golovinski P. A., Vitukhnovsky A. G. 2016. The size dependence recombination luminescence of hydrophilic colloidal CdS quantum dots in gelatin. Journal of Luminescence, 179: 413-419. 


\section{References}

1. Bateman H., Erdelyi A. 1953. Higher transcendental functions T. 1. New York, McGraw-Hill, 325. (in Russian)

2. Hobson E. W. 1931. The theory of spherical and ellipsoidal harmonics. Cambridge, Cambridge University Press, 500. (in Russian)

3. Katrakhov V. V., Sitnik S. M. 2018. Metod operatorov preobrazovaniya i kraevye zadachi dlya singulyarnykh ellipticheskikh uravnenii [Method of transformation operators and boundary value problems for singular elliptic equations]. Sovremennaya matematika. Fundamental'nye napravleniya, 64(2): 211-426.

4. Keldysh M. V. 1951. O nekotorykh sluchayakh vyrozhdeniya uravneniy ellipticheskogo tipa na granitse oblasti [On some structures of degenerate equations of elliptic type on the boundary of a region]. Doklady Academii Nauk SSSR, 77(2): 181-183.

5. Kipriyanov I. A. 1997. Singulyarnye ellipticheskie kraevye zadachi [Singular elliptic boundary value problems], M., Nauka.Fizmatlit, 208.

6. Landis E. M. 1971. Uravnenie vtorogo poryadka ellipticheskogo i parabolicheskogo tipov [Secondorder equations of elliptic and parabolic types]. M., Nauka, 288.

7. Larin A. A., Kirillov V. P. 2017. Zadacha na sobstvennye zhacheniya dlya odnogo obyknovennogo differencial'nogo operatora s singulyarnym koefficientom [The eigenvalue problem for one ordinary differential operator with a singular coefficient]. Sbornik trudov X mezhdunarodnoy konferent-sii «PMTUKT-2017» (Voronezh, 18-24 sentyabrya 2017 g.). Voronezh, Izd-vo Nauchnaya kniga, 221225.

8. Nikiphorov A. F., Uvarov V. B. 1984. Special'nye funkcii matematicheskoi fiziki [Special functions of mathematical physics]. M., Nauka, 344.

9. Titchmarsh E. C. 1958. Eigenfunction expansions associated with second-order equations Ch. 2. Oxford, Clarendon Press, 404. (in Russian)

10. Gavrilenko V. I. 2011. Optics of Nanomaterials. Singapore, Pan Stanford Publishing, 326.

11. Klingshirn C. 2005. Semiconductors Optics. Berlin, Springer-Verlag Heidelberg, 797.

12. Klimov V. I. 2010. Nanocrystal quantum dots. New York, CRC Press, 453.

13. Mora-Ramos M. E., Aouami A. El., Feddi E., Radu A., Restrepo R. L., Vinasco J. A., Morales A. L., Duque C. A. 2020. Donor impurity energy and optical absorption in spherical sector quantum dots. Heliyon, 6: e03194(11 pp).

14. Ovchinnikov O. V., Smirnov M. S., Korolev N. V., Golovinski P. A., Vitukhnovsky A. G. 2016. The size dependence recombination luminescence of hydrophilic colloidal CdS quantum dots in gelatin. Journal of Luminescence, 179: 413-419.

Королев Никита Викторович - кандидат физико-математических наук, доцент кафедры математики Военного учебно-научного центра Военно-воздушных сил «Военно-воздушная академия имени профессора Н. Е. Жуковского и Ю. А. Гагарина» (г. Воронеж) Министерства обороны Российской Федерации

ул. Ст. Большевиков, 54a, Воронеж, Россия,

E-mail: korolevn33@yandex.ru

Ларин Александр Александрович - кандидат физико-математических наук, доцент, доцент кафедры математики Военного учебно-научного центра Военно-воздушных сил «Веено-воздушная академия имени профессора Н. Е. Жуковского и Ю. А. Гагарина» (г. Воронеж) Министерства обороны Российской Федерации

ул. Ст. Большевиков, 54a, Воронеж, Россия,

E-mail: dohior@yandex.ru 